\title{
Clinical evaluation of the efficacy of oral amiodarone
}

\author{
WILLIAM J MCKENNA, DENNIS M KRIKLER \\ From the Division of Cardiovascular Disease, Department of Medicine, Royal Postgraduate Medical School, London
}

Amiodarone is an effective antiarrhythmic agent whose wider use has been limited by unwanted effects. ${ }^{1}$ As most of the troublesome and serious side effects are dose related it is particularly important to use the minimum effective maintenance dose, ${ }^{2}$ but this may be difficult to determine because of the pharmacological properties of amiodarone. Bioavailability in normal volunteers varies widely $(20-80 \%)$ and this must contribute to the wide range of dosage required to control a particular arrhythmia: from as little as $\mathbf{4 0 0} \mathrm{mg}$ weekly up to $\mathbf{8 0 0} \mathrm{mg}$ daily. In addition, the assessment of drug efficacy is complicated by the long elimination half life $\left(t^{1 / 2}\right)$ which is usually 30 to 60 days but which may be much greater particularly in obese patients. Evaluation of symptoms and electrocardiographic monitoring do not permit differentiations of drug failure from the need for additional amiodarone, nor do they identify those patients in whom arrhythmia may be controlled with less amiodarone. A clinical marker of drug efficacy is needed to help determine when to increase dosage if arrhythmia persists and when to decrease when arrhythmia is controlled.

The demonstration by Debbas and her colleagues ${ }^{3}$ of a strong correlation between the change in QT interval is thus timely. It is an indication of the difficulties inherent in treating such patients that this was possible in only nine of their group of 30 patients. These findings indicate that when reliable measurements can be made, which is only possible in the absence of other cardioactive medications, changes in QT interval may be a useful marker of myocardial impregnation and drug effect. Indeed in larger series others have found that the QT interval may not be so reliable. ${ }^{4}$ This is partly because new $U$.waves often appear either within the $T$ wave or isolated from it. ${ }^{5}$ Serial observations in individual patients may reveal

Requests for reprints to Dr W J McKenna, Division of Cardiovascular Disease, Hammersmith Hospital, Du Cane Road, London W12 OHS. only retrospectively that $\mathrm{QT}$ prolongation was due to incorporation of $U$ waves within the measurement. The significance of $U$ waves during treatment with amiodarone is uncertain; they have been observed to appear and disappear during treatment and they may obscure the precise end of the QT interval. In addition, increases in the QT interval are an insensitive marker of myocardial impregnation as patients demonstrate control of arrhythmia in the absence of QT prolongation and the correlation of antiarrhythmia effect and QT prolongation is poor. ${ }^{4}$ In isolated patients assessment of the QT interval provides valuable clinical information: shortening during amiodarone may indicate failure of compliance or the development of thyrotoxicosis; pronounced lengthening may identify those at increased risk of torsade de pointes. In the majority, however, measurement of the QT interval is not a useful guide to the identification of those who may benefit from alteration in dosage, and from the present work $^{3}$ one may achieve success in a maximum of one third of patients.

Others have suggested that reverse triiodothyronine levels are useful in monitoring efficacy and side effects. ${ }^{6}$ Amiodarone inhibits the peripheral conversion of thyroxine to triiodothyronine in favour of reverse triiodothyronine. During chronic treatment most patients will show an increase in thyroxine, a normal or low T3, and an increase in reverse T3 unaccompanied by alteration in thyroid state. Though an approximate therapeutic as well as toxic range of reverse T3 has been determined in a small group of patients who experienced suppression of arrhythmia during amiodarone, the role of this measurement in relation to dosage changes remains to be defined. Because it is influenced by stress, concurrent illness, and thyroid disease, reverse triiodothyronine will possibly not prove the reliable index we seek. In our investigations measurement of plasma drug concentrations has provided a valuable guide to the appropriateness and magnitude of dosage changes. Though a 
specific therapeutic range remains to be determined control of arrhythmias is most often achieved with plasma amiodarone and desethylamiodarone concentrations of $1.5 \mu \mathrm{g} / \mathrm{ml}$ or less whereas higher levels are associated with an increased incidence of unwanted effects. ${ }^{7}$ Thus when arrhythmia is controlled with low plasma concentrations it may be appropriate to reduce the daily dose by a small amount while a larger reduction is indicated when plasma levels are high. When arrhythmia is not controlled a low plasma level suggests reduced bioavailability and the requirement for an increased dose, while high plasma levels indicate the need for additional or alternative therapy. During long term amiodarone treatment of patients with hypertrophic cardiomyopathy and refractory arrhythmia incremental dosage changes were guided by electrocardiographic monitoring and measurement of plasma amiodarone concentrations; in striving to achieve the lowest effective maintenance dose serious side effects were rare and only one of 53 patients stopped taking amiodarone. ${ }^{8}$ This approach is feasible in the management of arrhythmias which are not highly malignant. Though the principle of achieving the lowest effective dose is equally valid, alternative methods are necessary in patients with ventricular tachycardia which is prone to degenerate to fibrillation or which has been associated with impaired consciousness. The role of programmed electrical stimulation in this regard has been extensively explored but is confused by conflicting results from major centres. ${ }^{9-11}$ Though the inducibility of ventricular tachycardia is not a predictor of subsequent efficacy, ${ }^{12} 13$ the characteristics of the arrhythmia may be so. ${ }^{10}$

At present then there is no adequate single measurement to guide alterations in treatment. The findings of Debbas et al indicate that in a pure group changes in the QT interval may be of clinical value. ${ }^{3}$ The strong correlation of plasma and myocardial amiodarone concentrations which they demonstrated suggests that plasma levels reflect myocardial impregnation and that when they are available they may be useful in guiding treatment.

\section{References}

1 Harris L, McKenna WJ, Rowland E, Holt DW, Storey
GCA, Krikler DM. Side effects of longterm amiodarone therapy. Circulation 1983; 67: 45-51.

2 McKenna WJ, Rowland E, Krikler DM. Amiodarone: the experience of the past decade. $B r$ Med $\mathcal{F} 1983 ; 287$ : $1654-6$.

3 Debbas NMG, du Cailar C, Bexton RS, Demaille JG, Camm AJ, Puech P. The QT interval: a predictor of the plasma and myocardial concentrations of amiodarone. $\mathrm{Br}$ Heart $\mathcal{F}$ 1984; 51: 316-20.

4 Rosenbaum MB, Chiale PA, Halpern MS, et al. Clinical efficacy of amiodarone as an antiarrhythmic agent. $A m \mathcal{F}$ Cardiol 1976; 38: 934-44.

5 Facquet PJ, Nivet M, Grosgogeat V, Alhomme P, Vachon J. L'influence de l'amiodarone sur le rythme cardiaque et l'electrocardiogramme. Therapie 1970; 25: 335-41.

6 Nademanee K, Singh BN, Hendrickson JA, Reed AW, Melmed S, Hershman J. Pharmacokinetic significance of serum reverse T3 levels during amiodarone treatment: the potential method for monitoring chronic drug therapy. Circulation 1982; 66: 202-11.

7 Haffajee CI, Love JC, Canada AT, Lesko LJ, Asdourian G, Alpert JS. Clinical pharmacokinetics and efficacy of amiodarone for refractory tachyarrhythmias. Circulation 1983; 67: 1347-55.

8 McKenna WJ, Rowland E, Harris L, Holt D, Krikler DM. Amiodarone treatment of refractory arrhythmia in hypertrophic cardiomyopathy: determination of the minimum effective dose [Abstract]. Circulation 1983; 68 (suppl): III-279.

9 Borggrefe M, Breithardt G, Seipel L. Value of serial electrophysiological testing in the treatment of ventricular tachyarrhythmias with amiodarone. Circulation 1983; 68 (suppl): III-381.

10 Horowitz LN, Spielman SR, Greenspan AN, Webb CR, Kay HR. Utility of electrophysiologic testing of amiodarone for ventricular tachyarrhythmias. Circulation 1983; 68 (suppl): III-382.

11 Waxman HL, Buxton AE, Marchlinski FE, Flores BT, Rogers DP, Josephson ME. Amiodarone for sustained ventricular tachyarrhythmias: electrophysiologic study is not predictive of clinical efficacy. Circulation 1983; 68 (suppl): III-382.

12 Heger JJ, Prystowsky EN, Jackman WM, et al. Amiodarone. Clinical efficacy and electrophysiology during long-term therapy for recurrent ventricular tachycardia or ventricular fibrillation. $N$ Engl f Med 1981; 305: $539-45$.

13 Roy D, Waxman HL, Kienzle MG, Buxton AE, Marchlinski FE, Josephson ME. Clinical characteristics and longterm follow up in 119 survivors of cardiac arrest: relation to inducibility at electrophysiologic testing. $\mathrm{Am}$ f Cardiol 1983; 52: 969-74. 